\title{
Covert symmetries in the neutrino mass matrix
}

\author{
Fredrik Björkeroth, ${ }^{a}$ Luca Di Luzio, ${ }^{b, c}$ Federico Mescia $^{d}$ and Enrico Nardi ${ }^{a}$ \\ ${ }^{a}$ INFN, Laboratori Nazionali di Frascati, \\ C.P. 13, 100044 Frascati, Italy \\ ${ }^{b}$ Deutsches Elektronen-Synchrotron DESY, \\ Notkestraße 85, D-22607 Hamburg, Germany \\ ${ }^{c}$ Dipartimento di Fisica, Università di Pisa and INFN - Sezione di Pisa, \\ Largo B. Pontecorvo 3, 56127 Pisa, Italy \\ ${ }^{d}$ Departament de Fúsica Quàntica i Astrofísica, Institut de Ciències del Cosmos (ICCUB), \\ Universitat de Barcelona, Martí Franquès 1, E08028 Barcelona, Spain \\ E-mail: fredrik.bjorkeroth@gmail.com, luca.diluzio@desy.de, \\ mescia@ub.edu, enrico.nardi@lnf.infn.it
}

ABSTRACT: The flavour neutrino puzzle is often addressed by considering neutrino mass matrices $m$ with a certain number of vanishing entries $\left(m_{i j}=0\right.$ for some values of the indices), since a reduction in the number of free parameters increases the predictive power. Symmetries that can enforce textures zero can also enforce a more general type of conditions $f\left(m_{i j}\right)=0$ with $f$ some function of the matrix elements $m_{i j}$. In this case $m$ can have all entries non-vanishing with no reduction in its predictive power. We classify all generation-dependent U(1) symmetries which, in the presence of two leptonic Higgs doublets, can reduce the number of independent high-energy parameters of type-I seesaw to the minimum number compatible with non-vanishing neutrino mixings and $\mathrm{CP}$ violation. These symmetries are broken above the scale where the effective operator is generated and can thus remain covert, in the sense that no explicit evidence of the symmetry can be read off the neutrino mass matrix, and different symmetries can give rise to the same low-energy structure. We find that only two cases are viable: one yields a structure with two zero-textures already considered in the literature, the other has no zero-textures and has never been considered before. It predicts normal ordering, a lightest neutrino mass $\sim 10 \mathrm{meV}$, a Dirac phase $\delta \sim \frac{3 \pi}{2}$ and definite values for the Majorana phases.

Keywords: Global Symmetries, Neutrino Physics, Beyond Standard Model

ARXIV EPRINT: 1910.00576 


\section{Contents}

1 Introduction 1

2 General considerations 2

3 Minimal seesaw models $\quad 5$

$\begin{array}{lll}3.1 & \text { Neutrino mass structures } & 7\end{array}$

$\begin{array}{lll}3.2 & \text { Matrix textures and analytical constraints } & 10\end{array}$

4 Confronting textures with neutrino data $\quad 12$

4.1 Analytical expressions for the Majorana phases 12

$\begin{array}{lll}4.2 & \text { Numerical analysis } & 14\end{array}$

$\begin{array}{lll}4.3 & \text { Results } & 15\end{array}$

$\begin{array}{lll}5 & \text { Concluding remarks } & 17\end{array}$

A $\mathrm{U}(1)$ charges for viable neutrino mass textures $\quad 20$

$\begin{array}{ll}\text { B Signature of the eigenvalues for texture D } & 21\end{array}$

\section{Introduction}

Flavour symmetry groups have been widely used in attempts to account for the mass and mixing patterns of the Standard Model (SM) quarks and leptons. Fermions are often assumed to transform in representations of elementary continuous groups like U(1) [110], $\mathrm{U}(2)$ [11-15], $\mathrm{SU}(3)$ [16-19], spontaneously broken by the vacuum expectation values (vevs) of scalar fields which are generally singlets of the SM or in the adjoint of some GUT group [20-22]. The symmetry and symmetry-breaking patterns are usually chosen in such a way that the symmetric limit reproduces the gross features of the fermion spectrum (for example only the top quark or the third family of charged fermions acquire mass) while corrections proportional to the symmetry-breaking vevs account for a parametric suppression of numerically small quantities (e.g. light fermion masses and inter-generational quark mixings). In the charged fermion sector this strategy is well justified by the presence of a certain number of $O(1)$ parameters that are naturally assumed to be non-vanishing in the symmetric limit. However, because of the exceedingly small value of neutrino masses, it is conceivable that the whole structure of the neutrino sector might depend solely on symmetry-breaking effects, in which case it would be hard to identify a flavour symmetry, even when accurate determinations of all the relevant parameters (neutrino masses, mixing, and $\mathrm{CP}$ violating phases) will be available. ${ }^{1}$

\footnotetext{
${ }^{1}$ The opposite situation in which the symmetric limit provides a good approximation to the structure of the neutrino mass matrix has been recently thoroughly analysed in [23].
} 
As is well known, an elegant explanation for the tininess of neutrino masses relies on the assumption that they arise from a non-renormalizable effective operator of dimension five, suppressed by some large scale. Then, if a flavour symmetry exists, but it is broken above the scale at which the effective operator is generated, it can remain covert in the structure of the effective mass operator. As we will show, it is however possible that far-reaching consequences of the symmetry survive in the effective theory in the form of non-trivial (although possibly complicated) relations between low-energy observables. In this paper we study this possibility in some detail. We assume that the neutrino mass operator is generated by the type-I seesaw mechanism with three right-handed $(\mathrm{RH})$ neutrinos, and that the form of the high-energy renormalizable Lagrangian is determined by a simple U(1) flavour symmetry with generation-dependent charges. We assume that the symmetry is spontaneously broken by the vev $f_{\phi}$ of a SM singlet field $\phi$, which in our construction is the only new field besides one additional Higgs doublet and the three RH neutrinos. The latter acquire their masses from this breaking, hence $f_{\phi}$ is the only high-energy scale.

Much in the spirit of the study carried out in ref. [24] for the quark sector, we search for $\mathrm{U}(1)$ charge assignments that can reduce the number of independent parameters to the minimum number compatible with non-vanishing lepton masses, neutrino mixings, and $\mathrm{CP}$ violation, and we argue that such constructions necessarily involve at least two Higgs doublets. In the quark sector it was found that this number matches exactly the number of SM observables [24], resulting in a complete determination of the model parameters, but no predictions for the SM observables. For the lepton sector we find instead a different result. In order to match our phenomenological requirements, the high-energy Yukawa sector must involve at least nine real plus one complex parameter. However, after integrating out the $\mathrm{RH}$ neutrinos, the number of real parameters needed to describe the charged lepton (CL) masses and the effective neutrino mass matrix is reduced to six. Thus, in our construction, the twelve physical observables of the lepton sector (three CL and three neutrino masses, three mixing angles and three CP-violating phases) turn out to be related by four nontrivial conditions that are the low-energy consequences of the original flavour symmetry.

The paper is structured as follows. In section 2 we introduce the basic theoretical framework. In section 3 we classify the $\mathrm{U}(1)$ flavour models in terms of different types of mass matrices which are 'minimal' in the sense described above (the three high-energy matrices have all together only ten non-vanishing entries) and we derive the relations between low-energy observables that are the consequence of the flavour symmetry. In section 4 we confront various textures with the experimental data, identify the viable possibilities, and assess their predictions. In section 5 we summarise our findings and draw the conclusions. Proofs and ancillary results are given in two appendices.

\section{General considerations}

A Majorana mass matrix for the light neutrinos can be described by a $D=5$ effective Weinberg operator, generated at some large scale $\Lambda_{L} \gg \Lambda_{\mathrm{EW}}$ (with $\Lambda_{\mathrm{EW}}$ the electroweak breaking scale) that is the scale suppressing neutrino masses and lepton number $(L)$ nonconserving processes. We assume that the renormalizable high-energy couplings of the 
uncoloured matter fields respect a generation-dependent $\mathrm{U}(1)$ symmetry which is spontaneously broken by a large vev $f_{\phi} \gg \Lambda_{\mathrm{EW}}$ of a SM singlet scalar field $\phi .^{2}$ We have two possibilities:

1) $f_{\phi}<\Lambda_{L}$ : the U(1) symmetry is still a good symmetry in the effective theory after $L$-breaking. $D=5$ operators which respect $U(1)$ are initially generated. After $\mathrm{U}(1)$ (and EW) symmetry breaking the structure of the mass matrix will still reflect the underlying symmetry in some approximation, e.g. entries that are forbidden in the symmetric limit will remain suppressed.

2) $f_{\phi}>\Lambda_{L}$ : the $\mathrm{U}(1)$ symmetry is broken already at the level of the renormalizable Lagrangian. Since there is no symmetric limit for the low-energy effective operators, their structure cannot directly hint to the U(1) symmetry. Still, the mass matrices can inherit from the initial symmetry a set of nontrivial relations among their entries.

In this paper we explore the second possibility. For definiteness we work in the framework of type-I seesaw with three $\mathrm{RH}$ neutrinos, with $\Lambda_{L}$ corresponding to the $\mathrm{RH}$ neutrino mass scale. ${ }^{3}$ We assume that $f_{\phi}$ is the only high-energy scale, and that the $\mathrm{RH}$ neutrino masses are generated by the $\mathrm{U}(1)$-breaking vev as $\Lambda_{L}=\lambda f_{\phi}$, with $\lambda$ a generic Yukawa coupling. For $\lambda \lesssim 1$ the condition $\Lambda_{L} \lesssim f_{\phi}$ is then naturally realised. As a guiding principle, we require that the $\mathrm{U}(1)$ symmetry enforces a maximal reduction in the number of free parameters compatible with the qualitative requirements of non-vanishing CL and neutrino masses, non-vanishing neutrino mixings, and $\mathrm{CP}$ violation. While a vanishing mass for the lightest neutrino is still not ruled out, and CP conservation in the lepton sector is still allowed by present data [26, 27], we think that neither of these possibilities has strong theoretical motivation and, accordingly, we will not discuss these scenarios.

We denote the RH CL as $e_{\alpha}$, the RH heavy neutrinos as $N_{\alpha}$, and the left-handed (LH) lepton doublets as $\ell_{\alpha}=(\tilde{\nu}, e)_{\alpha}^{T}$, with $\alpha$ a generation index. ${ }^{4}$ To define a flavour symmetry that can act in the most general possible way, regardless of the type of field $(N, \ell$ or $e)$ and of the generation index, we need to introduce at least two Higgs doublets $H_{1,2}$ with the same weak hypercharge $Y=+1 / 2$ but with different $\mathrm{U}(1)$ charges $\mathcal{X}\left(H_{1}\right)=\mathcal{X}_{1}$ and $\mathcal{X}\left(H_{2}\right)=\mathcal{X}_{2} \neq \mathcal{X}_{1}$. Since all $\mathrm{U}(1)$ charges can be freely redefined by a shift proportional to the hypercharge of the corresponding field, we can set $\mathcal{X}_{1}=-\mathcal{X}_{2}$. To explicitly break the $\mathrm{U}(1)^{3}$ rephasing symmetry of the kinetic terms of the three scalars down to $\mathrm{U}(1)^{2}$

\footnotetext{
${ }^{2}$ This symmetry could be either local or global. In order to avoid complications with gauge anomalies, we assume for simplicity a global U(1).

${ }^{3}$ Type-II seesaw models introduce an SU(2) triplet $\Delta$ together with a Yukawa term $\bar{L}^{c} \Delta L$. The strategy of parameter reduction that we follow in our construction is not viable in the the minimal model with a single triplet, but might be viable with two or more scalar triplets. In the type-III seesaw the singlet $\mathrm{RH}$ neutrinos are replaced by $\mathrm{SU}(2)$ triplet fermions. In this case our strategy can be straightforwardly implemented along the same lines discussed here, and would yield similar results. It remains to be seen if analogous constructions can be implemented in models where the Weinberg operator is generated via loop diagrams [25].

${ }^{4} \mathrm{~A}$ tilde over the neutrino fields $(\tilde{\nu})$ refers to the neutrinos in the basis in which the CL carry well-defined $\mathrm{U}(1)$ charges, $\nu_{\alpha}(\alpha=e, \mu, \tau)$ denotes the neutrino combinations that couple to the CL mass eigenstates and defines the 'neutrino flavor basis', $\nu_{i}^{\prime}(i=1,2,3)$ refers to neutrino mass eigenstates.
} 
corresponding to hypercharge $\times$ flavour $\mathrm{U}(1)$, a non-Hermitian coupling between $H_{1,2}$ and $\phi$ is needed. There are two inequivalent possibilities corresponding to the two renormalizable operators:

$$
H_{1}^{\dagger} H_{2} \phi, \quad H_{1}^{\dagger} H_{2} \phi^{2} .
$$

We normalize the charges by choosing $\mathcal{X}_{\phi}=2$ so that we can label the two cases by the two different values of the Higgs charges, namely: Case 1: $\left|\mathcal{X}_{1,2}\right|=1$ and Case 2: $\left|\mathcal{X}_{1,2}\right|=2$. To avoid bare EW-invariant mass terms we assume that there are no $\mathrm{U}(1)$-invariant $\mathrm{RH}$ neutrino bilinears, i.e. $\mathcal{X}\left(N_{\alpha} N_{\beta}\right) \neq 0$. The renormalizable seesaw Lagrangian can then be written as

$$
\mathcal{L}=-\bar{e} \lambda^{e} \ell H_{n}-\bar{N} \lambda^{\nu} \ell \tilde{H}_{n}-\frac{1}{2} \overline{N^{c}} \lambda^{N} N \phi^{(*)}+\text { h.c. },
$$

where $\lambda^{e}$ and $\lambda^{\nu}$ are generic non-singular Yukawa matrices of complex couplings, $\lambda^{N}$ is non-singular complex symmetric, $H_{n}=H_{1}$ or $H_{2}$ as determined by U(1) invariance, and similarly $\phi^{(*)}=\phi$ or $\phi^{*}$. Note that depending on charge assignments, for each one of the three terms in eq. (2.2), some couplings can be forbidden, and will correspond to zero entries in the Yukawa matrices. Let us introduce the mass matrices

$$
m_{e}=\lambda^{e} v_{n}, \quad m_{D}=\lambda^{\nu} v_{n}, \quad M_{N}=\lambda^{N} f_{\phi}, \quad \tilde{m}_{\nu}=-m_{D}^{T} M_{N}^{-1} m_{D},
$$

where $v_{n}=v_{1}$ or $v_{2}$ with $v_{1,2}=\left\langle H_{1,2}\right\rangle$ the EW breaking vevs, $f_{\phi}=\langle\phi\rangle$, and $\tilde{m}_{\nu}$ is the mass matrix for the light neutrinos generated by the seesaw mechanism. The matrices $m_{e}$ and $\tilde{m}_{\nu}$ can be written in terms of the singular values $\hat{m}_{e}=\operatorname{diag}\left(m_{e}, m_{\mu}, m_{\tau}\right)$ and $\hat{m}_{\nu}=\operatorname{diag}\left(m_{1}, m_{2}, m_{3}\right)$ as: ${ }^{5}$

$$
\begin{aligned}
& m_{e}=R \hat{m}_{e} L^{\dagger}, \\
& \tilde{m}_{\nu}=(V \Phi)^{*} \hat{m}_{\nu}(V \Phi)^{\dagger},
\end{aligned}
$$

where $R, L$ and $V$ are $\mathrm{SU}(3)$ matrices and $\Phi$ is a diagonal matrix of phases chosen in such a way that the singular values of $\tilde{m}_{\nu}$ are real and positive. The mass eigenstate basis for the LH charged leptons and for the neutrinos is defined respectively as $e_{L}^{\prime}=L^{\dagger} e_{L}$ and $\nu^{\prime}=(V \Phi)^{\dagger} \tilde{\nu}$, and the charged current interaction then reads

$$
\mathcal{L}_{C C}=\frac{g}{\sqrt{2}} \overline{e_{L}} \gamma^{\mu} \tilde{\nu} W_{\mu}^{-}+\text {h.c. }=\frac{g}{\sqrt{2}} \bar{e}_{L}^{\prime} \gamma^{\mu} U \nu^{\prime} W_{\mu}^{-}+\text {h.c. },
$$

where

$$
U=L^{\dagger} V \Phi
$$

is the lepton mixing matrix that defines the particular combination $\nu_{\alpha}=U_{\alpha i} \nu_{i}^{\prime}$ of the three neutrino mass eigenstates $\nu_{i}^{\prime}$ that couples to a specific CL $\left(e_{L}^{\prime}\right)_{\alpha}$ of flavour $\alpha=e, \mu, \tau$. The basis $\left(\nu_{e}, \nu_{\mu}, \nu_{\tau}\right)$ is generally referred as the 'neutrino flavour basis', and here it represents a third basis for the neutrino fields. (The basis of mass eigenstates and the basis in which the CL fields carry well-defined U(1) charges have been introduced in footnote 4.) The effective neutrino mass matrix written in this basis reads

$$
m_{\nu}=L^{T} \tilde{m}_{\nu} L=U^{*} \hat{m}_{\nu} U^{\dagger} .
$$

\footnotetext{
${ }^{5}$ We will commonly denote these singular values as 'mass eigenvalues'.
} 
We will parametrise the neutrino masses in terms of an overall scale $\mu_{0}$ corresponding to the mass of the lightest neutrino, and of the two mass-squared differences $\Delta_{\odot}$ (solar) and $\Delta_{\oplus}$ (atmospheric) which have been measured with fair precision in oscillation experiments. For $\mu_{0}$ only upper bounds exist, the most stringent of which comes from cosmological considerations. This bound is expressed as an upper limit on the the sum of the three neutrino masses, and reads $\sum_{i} m_{i} \lesssim 0.12-0.60 \mathrm{eV}$, depending on which set of data is used [28], however, for the purpose of ruling out some types of neutrino mass matrix, it will be sufficient for us to impose the much looser bound $\mu_{0} \lesssim 1 \mathrm{eV}$. The mass ordering is also not fully determined: we know the sign of the solar mass square difference, and we know that $\left|\Delta_{\odot}\right| \ll\left|\Delta_{\oplus}\right|$, but the sign of the atmospheric mass difference is yet unknown. Accordingly, there are two possible orderings for neutrino masses: $\mu_{0}=m_{1}<m_{2}<m_{3}$ which is denoted as normal ordering (NO), and $\mu_{0}=m_{3}<m_{1}<m_{2}$ which is denoted as inverted ordering (IO). ${ }^{6}$ The solar mass squared difference is conventionally defined as $\Delta_{\odot}=m_{2}^{2}-m_{1}^{2}>0$ and, by defining $\Delta_{\oplus}$ to be also positive, we have $\Delta_{\oplus}=m_{3}^{2}-m_{1}^{2}$ for $\mathrm{NO}$ and $\Delta_{\oplus}=m_{2}^{2}-m_{3}^{2}$ for IO, that is:

$$
\hat{m}_{\nu}^{2} \equiv \operatorname{diag}\left(m_{1}^{2}, m_{2}^{2}, m_{3}^{2}\right)= \begin{cases}\operatorname{diag}\left(\mu_{0}^{2}, \mu_{0}^{2}+\Delta_{\odot}, \mu_{0}^{2}+\Delta_{\oplus}\right) & (\mathrm{NO}), \\ \operatorname{diag}\left(\mu_{0}^{2}+\Delta_{\oplus}-\Delta_{\odot}, \mu_{0}^{2}+\Delta_{\oplus}, \mu_{0}^{2}\right) & (\mathrm{IO}) .\end{cases}
$$

\section{Minimal seesaw models}

Generic seesaw models for lepton masses and mixings usually introduce a number of free parameters much larger than the number of low-energy observables. In this respect, models that only involve the minimum number of parameters needed to ensure compatibility with the observed phenomenology are quite economical, and can thus be considered particularly attractive. Following the same strategy adopted in ref. [24] to search for minimal models for the quark sector, we assume that parameter reduction in the seesaw Yukawa Lagrangian (eq. (2.2)) is enforced by a global $\mathrm{U}(1)$ symmetry that acts on the fermionic fields $\ell, e, N$ and on the scalars $H_{1,2}$ and $\phi$, and that forbids the maximum number of entries in the Yukawa matrices $\lambda^{e}, \lambda^{\nu}, \lambda^{N}$ compatibly with the following requirements:

1. There are no U(1)-invariant bilinears of the $\mathrm{RH}$ neutrino fields, so that $M_{N}$ depends on a single high-energy scale $f_{\phi}$. This implies $\mathcal{X}\left(N_{\alpha}\right) \neq 0$ and $\mathcal{X}\left(N_{\alpha}\right) \neq-\mathcal{X}\left(N_{\beta}\right)$ for all $\alpha, \beta=1,2,3$.

2. All fermions, including the three light neutrinos, are massive, hence $\lambda^{e}, \lambda^{\nu}, \lambda^{N}$ and the corresponding mass matrices $m_{e}, m_{D}, M_{N}$ in eq. (2.3) have nonzero determinant. This also ensures $\operatorname{det} m_{\nu} \neq 0$.

3. The leptonic Jarlskog invariant $J=\operatorname{Im}\left[U_{11} U_{13}^{*} U_{31}^{*} U_{33}\right]$ is non-vanishing. This is equivalent to require that all the neutrino mixing angles are non-zero and that the Dirac CP violating phase is non vanishing, and implies that at least one complex Yukawa coupling cannot be made real by field redefinitions.

\footnotetext{
${ }^{6}$ Recent fits to the neutrino data $[26,27,29-31]$ indicate a preference for NO at about $3 \sigma$.
} 
4. The way the zero and non-zero entries are arranged in the mass matrices must be consistent with some U(1) symmetry, i.e. it must be possible to enforce the corresponding structures by assigning to the fields a complete set of $\mathrm{U}(1)$ charges.

It is straightforward to obtain the minimum number of non-vanishing entries in the three Yukawa matrices in eq. (2.2) (or equivalently in $m_{e}, m_{D}, M_{N}$ in eq. (2.3)) that are required to satisfy the second and third requirements. To ensure non-vanishing determinants we need at least three non-vanishing entries in both $m_{e}$ and $m_{D}$, which can be taken to lie on the diagonal by a suitable labeling of the fields. For $M_{N}$, which is symmetric, two parameters (three non-zero entries) suffice when they are taken for example as in $M_{N}^{(2)}$ in eq. (3.1). In this case we need two off-diagonal entries in $m_{e}$ and/or $m_{D}$ to generate all the mixings, for a total of ten parameters. Alternatively, $M_{N}$ may involve three parameters (five non-zero entries) as in the matrix $M_{N}^{(3)}$ in eq. (3.1),

$$
M_{N}^{(2)} \sim\left(\begin{array}{ccc}
\times & 0 & 0 \\
0 & 0 & \times \\
0 & \times & 0
\end{array}\right), \quad M_{N}^{(3)} \sim\left(\begin{array}{ccc}
\times & \times & 0 \\
\times & 0 & \times \\
0 & \times & 0
\end{array}\right) .
$$

In the latter case one off-diagonal entry in $m_{e}$ or in $m_{D}$ suffices to ensure non-vanishing mixings, again for a total of ten parameters. It is easy to verify that in both these cases field redefinitions allow us to remove all but one complex phase, so that $J \neq 0$. We thus conclude that the minimum number of fundamental parameters required to construct highenergy type-I seesaw matrices qualitatively compatible with the SM lepton sector (in the sense that conditions 2 and 3 are satisfied) is ten. It is straightforward to verify that, not to exceed this number, the following condition must be also satisfied:

5. No pair of $N$ 's and no pair of $\ell$ 's are allowed to have the same charge.

This is because otherwise $2 \times 2$ blocks in a pair of matrices (respectively $M_{N}$ and $m_{D}$ or $m_{D}$ and $m_{e}$ ) will unavoidably fill up, implying a total of at least 12 parameters. In particular, this excludes the possibility of having more than three parameters in $M_{N}$. In contrast, a pair of RH leptons with equal charges will produce a non-vanishing $2 \times 2$ block only in $m_{e}$, so that parameter counting by itself does not exclude this possibility. Condition 5 then explains why at least two Higgs doublets are needed to implement schemes with a minimal number of parameters. Non vanishing lepton mixings require that some bilinears involving two different $\ell_{\alpha}$ in the first and/or second term in eq. (2.2) couple to a scalar doublet which develops a non vanishing vev. With a single Higgs, this would imply charge relations like $\mathcal{X}\left(\bar{e}_{\alpha} \ell_{\beta}\right)=\mathcal{X}\left(\bar{e}_{\alpha} \ell_{\gamma}\right)$ with $\beta \neq \gamma$, and hence $\mathcal{X}\left(\ell_{\beta}\right)=\mathcal{X}\left(\ell_{\gamma}\right)$.

In the following we will derive a complete classification of the neutrino mass matrix textures that are consistent with the requirement of minimality, and comply with the conditions 1-5 listed above. This program is carried out in three steps: first we discuss the possible ways to assign the ten fundamental parameters to the matrices $M_{N}, m_{D}$ and $m_{e}$. As a second step, for each possible assignment we study which are the viable structures for the neutrino mass matrix. We use the term structure to denote the equivalence class containing all those textures that can be transformed one into another by permutations of the row and column indices. As the third and final step we count how many inequivalent 


\begin{tabular}{|ccccc|}
\hline$M_{N}$ & $m_{D}$ & $m_{e}$ & & \\
\hline 2 & 5 & 3 & $\boldsymbol{x}$ & $\mathrm{U}(1)$ \\
2 & 3 & 5 & $\boldsymbol{x}$ & $\theta_{i j} \neq 0$ \\
2 & 4 & 4 & $\mathfrak{\checkmark}$ & $\left|\mathcal{X}_{1,2}\right|=1,2$ \\
3 & 4 & 3 & $\sqrt{ }$ & $\left|\mathcal{X}_{1,2}\right|=2$ \\
3 & 3 & 4 & $\sqrt{ }$ & $\left|\mathcal{X}_{1,2}\right|=1,2$ \\
\hline
\end{tabular}

Table 1. Possible ways to assign the ten parameters of a minimal type-I seesaw model to the mass matrices. The fourth column indicates which parameter assignments can be consistent with a U(1) symmetry and with three non-vanishing mixings. The last column indicates for the non viable cases which condition is violated, and for the remaining cases for which values of the Higgs charges they are viable.

textures correspond to each structure. As will become clear below, here the term texture will have a more general meaning than in its common use, since besides referring to the possible presence of vanishing elements, it will also refer to the presence of specific relations among different entries.

A clarification is in order regarding the notation that will be used below: neutrino mass matrix structures (and textures) only represent specific relations that must be satisfied by their elements, without any reference to numerical values for the non-vanishing entries, which remain unspecified. These structures/textures will be denoted by the symbol $\tilde{m}$ for the basis in which the CL U(1) charges are well-defined and $m_{e}$ in general is non-diagonal, and by the symbol $m$ for the basis in which the CL mass matrix is diagonal. This notation keeps the same meaning as for the matrices $\tilde{m}_{\nu}$ and $m_{\nu}$ already introduced (see eq. (2.7)), although the subscript " $\nu$ " is dropped. The subscripted form, $m_{\nu}$, will instead refer to the matrix containing experimental numbers. More precisely, $m_{\nu}=U^{*} \hat{m}_{\nu} U^{\dagger}$ is obtained by multiplying the experimentally determined PMNS mixing matrix by the diagonal matrix of the neutrino mass eigenvalues $\hat{m}_{\nu}$ which, for the two cases of normal or inverted ordering (see eq. (2.8)) encodes the available experimental information on neutrino masses. In short, there are several matrices $m$, one for each texture, that have to be confronted with $m_{\nu}(\mathrm{NO})$ or $m_{\nu}(\mathrm{IO})$ to asses whether they can be phenomenologically viable.

\subsection{Neutrino mass structures}

The possible ways of distributing the ten parameters among $M_{N}, m_{D}$ and $m_{e}$ while ensuring non-vanishing determinants for all three matrices, are listed in table 1. The first possibility in the first line is excluded because there is no way of assigning $\mathrm{U}(1)$ charges to $N_{\alpha}$ and $\ell_{\alpha}$ consistently with the structure of $M_{N}^{(2)}$ in such a way that $m_{D}$ has five non-vanishing entries. The second possibility can be implemented consistently with the U(1) symmetry but necessarily yields some vanishing mixing. This is because the form of $M_{N}^{(2)}$ eq. (3.1) implies that $m$ has two degenerate eigenvalues, and thus there are unphysical mixings. The other three possibilities are viable, although the one on the fourth line is consistent with $\mathrm{U}(1)$ only in case $\left|\mathcal{X}_{1,2}\right|=2$. For each viable configuration listed in table 1 we now explore the specific form of the viable textures. To denote the different possibilities it is convenient to introduce the following compact notation: each case will be labeled with a string of 
the form $\mathcal{X}(n)[i j][k l] . \mathcal{X}=\left|\mathcal{X}_{1,2}\right|=1,2$ distinguishes the two cases corresponding to the scalar couplings in eq. (2.1); $n=2,3$ denotes respectively the structure of the RH neutrino matrix $M_{N}^{(2)}$ or $M_{N}^{(3)}$ in eq. (3.1); the indices $i, j=1,2,3(i \neq j)$ within the first pair of square brackets denote which off-diagonal entry in $m_{D}$ is different from zero $\left(m_{D}^{i \neq j} \neq 0\right)$; $k, l$ in the second pair of brackets have the same meaning but for the charged lepton mass matrix $\left(m_{e}^{k \neq l} \neq 0\right)$. Furthermore, a pair of empty square brackets [ ] refers to a diagonal matrix, while indices within curly brackets refer collectively to both orderings $\{i j\}=[i j]$ or $[j i]$. For example:

$$
1(2)[12]\{12\}: \quad \mathcal{X}=1, \quad M_{N}=M_{N}^{(2)}, \quad m_{D} \sim\left(\begin{array}{ccc}
\times & \times & 0 \\
0 & \times & 0 \\
0 & 0 & \times
\end{array}\right), \quad m_{e} \sim\left(\begin{array}{ccc}
\times & \times & 0 \\
0 & \times & 0 \\
0 & 0 & \times
\end{array}\right) \text { or }\left(\begin{array}{ccc}
\times & 0 & 0 \\
\times & \times & 0 \\
0 & 0 & \times
\end{array}\right) .
$$

Deriving all the possible textures for the viable parameter assignments given in table 1 is a bit lengthy, but straightforward. It requires solving for the conditions $\left|\mathcal{X}\left(N_{i}\right)+\mathcal{X}\left(N_{j}\right)\right|=$ $\mathcal{X}_{\phi}=2,\left|\mathcal{X}\left(\bar{N}_{i}\right)+\mathcal{X}\left(\ell_{j}\right)\right|=\mathcal{X},\left|\mathcal{X}\left(\bar{e}_{i}\right)+\mathcal{X}\left(\ell_{j}\right)\right|=\mathcal{X}$ for the specific number of nonvanishing parameters in each mass matrix $M_{N}, m_{D}, m_{e}$, while respecting the constraint given above at point 5 , and ensuring that the total number of non-vanishing entries does not exceed ten. It is worth noting that for $M_{N}^{(3)}$ the viable $\mathrm{RH}$ neutrino charge assignment is unique (modulo an overall sign): $\mathcal{X}(N)=(1,-3,5)$, and this renders the derivation of $\mathcal{X}(\ell)$ and $\mathcal{X}(e)$ that yield the viable textures of type $\mathcal{X}(3)[i j][k l]$ particularly simple. Conversely, the structure of $M_{N}^{(2)}$ only implies for the RH neutrino charges the condition $\mathcal{X}(N)=\left(1, \mathcal{X}\left(N_{2}\right),-\mathcal{X}\left(N_{2}\right) \pm 2\right)$. Obtaining all textures then requires solving the system of constraints for increasing values of $\left|\mathcal{X}\left(N_{2}\right)\right|$ until no more solutions are found.

The viable structures that result from this procedure are listed in table 2 . The first half of the table (structures $\mathbf{A}, \mathbf{B}, \mathbf{H}, \mathbf{G}$ ) correspond to the RH neutrino mass matrix $M_{N}^{(2)}$, which is symmetric under the exchange $N_{2} \leftrightarrow N_{3}$. This means that structures obtained by exchanging the $(2,3)$ labels of all the fields are also viable. They have not been listed in the table because this becomes redundant in view of the next step of the analysis, that will precisely deal with field relabeling. By contrast, there is no such symmetry for $M_{N}^{(3)}$, and thus no structure has been omitted in the second half of the table. In the third column we give the form of the matrix $m_{e}^{\dagger} m_{e}$ from which the structure of the LH CL mixing matrix $L$ (see eq. (2.7)) can be read off. It is important to remark that $L$ is either trivial (structures E, F) or it corresponds to a simple matrix containing a $2 \times 2$ unitary block. In the fourth column $\tilde{m}$ gives the structure of the neutrino mass matrix in the basis in which the CL charges are well-defined (non-diagonal $m_{e}$ ), while the fifth column gives the form of the neutrino mass matrix $m$ in the flavour basis, in which $m_{e}$ is diagonal (since for $\mathbf{E}$ and $\mathbf{F}$ the matrix $L$ is trivial, in the last two lines $\tilde{m}=m$ ). The column labeled "Viable" marks whether the structure is compatible $(\boldsymbol{V})$ or not $(\boldsymbol{X})$ with experimental data.

It is readily seen that case $\mathbf{H}$ is not viable since the structures of $m_{e}^{\dagger} m_{e}$ and of $m$ imply that one lepton flavour does not mix with the others and thus there are vanishing mixings. Structures of type $\mathbf{E}$ have two vanishing parameters in $m$. Neutrino matrices of this type have been thoroughly studied in the literature [32-41] and it is known that they can yield viable models. More precisely, it is possible to find suitable textures in the 


\begin{tabular}{|c|c|c|c|c|c|c|}
\hline & Label & $m_{e}^{\dagger} m_{e}$ & $\tilde{m}$ & $m$ & Viable & Comments \\
\hline A & $\mathcal{X}(2)[21]\{12\}$ & $\left(\begin{array}{ccc}\times & \times & 0 \\
\times & \times & 0 \\
0 & 0 & \times\end{array}\right)$ & $\left(\begin{array}{ccc}\times & 0 & \times \\
0 & 0 & \times \\
\times & \times & 0\end{array}\right)$ & $\left(\begin{array}{ccc}\times & \times & \times \\
\times & \times & \times \\
\times & \times & 0\end{array}\right)$ & $x$ & \\
\hline B & $\mathcal{X}(2)[12]\{12\}$ & $\left(\begin{array}{ccc}\times & \times & 0 \\
\times & \times & 0 \\
0 & 0 & \times\end{array}\right)$ & $\left(\begin{array}{ccc}\times & \times & 0 \\
\times & \times & \times \\
0 & \times & 0\end{array}\right)$ & $\left(\begin{array}{ccc}\times & \times & \times \\
\times & \times & \times \\
\times & \times & 0\end{array}\right)$ & $x$ & \\
\hline $\mathbf{H}$ & $1(2)\{23\}\{23\}$ & $\left(\begin{array}{ccc}\times & 0 & 0 \\
0 & \times & \times \\
0 & \times & \times\end{array}\right)$ & $\left(\begin{array}{ccc}\times & 0 & \times \\
0 & 0 & \times \\
\times & x & \times\end{array}\right)$ & $\left(\begin{array}{ccc}\times & 0 & 0 \\
0 & \times & \times \\
0 & \times & \times\end{array}\right)$ & $x$ & $\theta_{i j} \neq 0$ \\
\hline G & $2(2)[12][13]$ & $\left(\begin{array}{ccc}\times & 0 & \times \\
0 & \times & 0 \\
\times & 0 & \times\end{array}\right)$ & $\left(\begin{array}{ccc}\times & \times & 0 \\
\times & \times & \times \\
0 & \times & 0\end{array}\right)$ & $\left(\begin{array}{ccc}\times & \times & \times \\
\times & \times & \times \\
\times & \times & \times\end{array}\right)$ & $\checkmark$ & \\
\hline C & $1(3)[]\{13\}$ & $\left(\begin{array}{ccc}\times & 0 & \times \\
0 & \times & 0 \\
\times & 0 & \times\end{array}\right)$ & $\left(\begin{array}{ccc}\times & 0 & \times \\
0 & 0 & \times \\
\times & \times & x\end{array}\right)$ & $\left(\begin{array}{ccc}x & x & \times \\
\times & 0 & \times \\
\times & \times & \times\end{array}\right)$ & $x$ & \\
\hline D & $1(3)[]\{12\}$ & $\left(\begin{array}{ccc}\times & \times & 0 \\
\times & \times & 0 \\
0 & 0 & \times\end{array}\right)$ & $\left(\begin{array}{ccc}\times & 0 & \times \\
0 & 0 & \times \\
\times & \times & \times\end{array}\right)$ & $\left(\begin{array}{ccc}x & x & \times \\
\times & x & \times \\
\times & \times & \times\end{array}\right)$ & $\checkmark$ & \\
\hline $\mathbf{E}$ & $2(3)[21][]$ & $\left(\begin{array}{ccc}\times & 0 & 0 \\
0 & \times & 0 \\
0 & 0 & \times\end{array}\right)$ & $\left(\begin{array}{ccc}\times & 0 & \times \\
0 & 0 & \times \\
\times & \times & \times\end{array}\right)$ & $\left(\begin{array}{ccc}\times & 0 & \times \\
0 & 0 & \times \\
\times & \times & \times\end{array}\right)$ & $\checkmark$ & Refs. $[32,33]$ \\
\hline $\mathbf{F}$ & $2(3)[31][]$ & $\left(\begin{array}{ccc}\times & 0 & 0 \\
0 & \times & 0 \\
0 & 0 & \times\end{array}\right)$ & $\left(\begin{array}{ccc}x & x & x \\
\times & 0 & \times \\
x & x & x\end{array}\right)$ & $\left(\begin{array}{ccc}\times & x & \times \\
\times & 0 & \times \\
\times & x & x\end{array}\right)$ & $x$ & \\
\hline
\end{tabular}

Table 2. Possible matrix structures for the viable parameter assignments listed in table 1 . In the first two lines $\mathcal{X}=\left|\mathcal{X}_{1,2}\right|$ is left unspecified, meaning that the same textures are obtained in both cases $\mathcal{X}=1$ and $\mathcal{X}=2$. Structures of type $\mathbf{H}$ yield some vanishing mixing, and are thus ruled out by the condition $\theta_{i j} \neq 0$ as is indicated in the last column. Structures of type $\mathbf{E}$ with two vanishing parameters can yield viable models. This type of matrices has been already thoroughly analysed in the literature [32-41], and hence are omitted from our analysis.

sense of specific positions for the zero entries, and specific values for the four non-vanishing parameters that fit well the experimental values for the three mixing angles and two mass squared differences, giving predictions for the remaining parameters. Quantitative analysis of two-zero neutrino mass textures have been performed recently in refs. [32, 33], hence we omit this type of structure from our numerical analysis. Let us, however, remark that while published numerical studies generally assume specific textures or specific relations between neutrino mass entries [42] from the start, here we have identified in a top-down approach which $\mathrm{U}(1)$ symmetries can produce such textures or, in other words, we have pinpointed the complete UV seesaw models in terms of viable sets of U(1) charge assignments. For completeness we list them in appendix A. ${ }^{7}$ For the remaining structures in table 2, their viability (or not) has been assessed by means of the numerical analysis described in the next section.

\footnotetext{
${ }^{7}$ For structure $\mathbf{E}$ there is a one-to-one correspondence between a specific texture for $m$ and the highenergy seesaw mass matrices, that is there is a unique string label (e.g. 2(3)[21][] for the case in table 2) and a unique set of charges identifying each model. It is thus conceivable that the underlying U(1) symmetry could be identified solely from low-energy data. Hence these cases do not properly belong to the class of "covert" U(1) symmetries.
} 


\subsection{Matrix textures and analytical constraints}

There are two important points that must be addressed before proceeding with the numerical analysis. The first regards the number of free parameters in the low-energy effective theory and the relationships between matrix elements. The second concerns the number of textures, or equivalently of models, obtained from a given low-energy structure by permutations of rows and columns.

Let us address the first point. The high-energy seesaw model depends on ten parameters, but three $\mathrm{RH}$ neutrino masses are integrated out, so that the effective theory is defined by seven parameters only. In the flavour basis, three parameters correspond to the CL masses, so $m$ must have only four independent parameters. There are three different ways in which this condition can be realised: $(i)$ two elements of $m$ vanish, as for structures of type $\mathbf{E}$; (ii) only one element vanishes, as for $\mathbf{A}, \mathbf{B}, \mathbf{C}, \mathbf{F}$, and thus one relation $f\left(m_{i j}\right)=0$ between the non-vanishing entries must hold; (iii) all elements of $m$ are non-vanishing, as for $\mathbf{G}, \mathbf{D}$, and then two relations must hold. The following results are derived by starting from an explicit form for $M_{N}$ and $m_{D}$ in terms of symbolic entries, deriving $\tilde{m}$ according to the last relation in eq. (2.3), writing down $m=L^{T} \tilde{m} L$ where the nontrivial $2 \times 2$ block in $L$ is written as a generic $\mathrm{SU}(2)$ matrix, and finally inspecting the resulting expression for $m$ to identify the sought relations.

Structures of type $\mathbf{E}$ have four non-vanishing entries that are therefore all independent. We can rephrase this by stating that textures belonging to class $\mathbf{E}$ must satisfy the two conditions

$$
(\mathbf{E}):\left\{\begin{array}{l}
m_{\bar{k} \bar{k}}=0, \\
m_{\bar{k} j}=0, \quad(j \neq \bar{k}),
\end{array}\right.
$$

where the barred index $\bar{k}$ must match between the two equations. This index-matching implies that, within our minimal scenario defined by $\mathrm{U}(1)$ charge consistency and two leptonic Higgs doublets, the two texture zeros must appear in the $2 \times 2$ block with rows and columns $j$ and $\bar{k}$. This excludes for example putting the zeros in the anti-diagonal, and analogous textures obtained by index permutations, which instead have been regularly considered in the literature on two-texture zero matrices.

In the flavour basis, structures of type $\mathbf{A}$ and $\mathbf{B}$ are the same, and $\mathbf{F}$ is equal to $\mathbf{C}$. Moreover the latter two can be obtained from the former by permuting the $(2,3)$ indices. These structures have a single (diagonal) zero entry, and thus, as anticipated, there must be one non-trivial relation between the other non-vanishing entries. Analysing the expression of $m$ in terms of explicit seesaw parameters this relation is easily derived, and it corresponds to the second of the following two conditions:

$$
(\mathbf{A}, \mathbf{B}, \mathbf{C}, \mathbf{F}):\left\{\begin{aligned}
m_{\bar{k} \bar{k}} & =0, \\
m_{i i} m_{j j}-m_{i j}^{2} & =0, \quad(i, j \neq \bar{k}),
\end{aligned}\right.
$$

where $\bar{k}$ must be chosen to match the unmixed entry in the CL mass matrix. Finally, structures $\mathbf{G}$ and $\mathbf{D}$, which are related by permutation of the $(2,3)$ indices (see table 2 ), have no zero entries, and thus there must be two non trivial relations between their six 
non-vanishing parameters. These two conditions are:

$$
(\mathbf{G}, \mathbf{D}):\left\{\begin{aligned}
m_{i i} m_{j j}-m_{i j}^{2} & =0, \\
\sum_{\ell=i, j}\left(m_{\ell \bar{k}}^{*} \sqrt{m_{\ell \ell} m_{\bar{k} \bar{k}}}-\left|m_{\ell \ell} m_{\bar{k} \bar{k}}\right|\right) & =0, \quad(i, j \neq \bar{k}),
\end{aligned}\right.
$$

where again $\bar{k}$ must match the index of the unmixed entry in the CL mass matrix $(\bar{k}=2$ for $\mathbf{G}$ and $\bar{k}=3$ for $\mathbf{D}$ ).

Let us now briefly comment on the number of independent CP-violating rephasing invariants. A generic $3 \times 3$ Majorana neutrino mass matrix has three independent invariants [43] that are usually expressed in terms of elements of the PMNS mixing matrix, but that can be also expressed in terms of elements of the effective neutrino mass matrix $m$ [44]. We focus here on case $(\mathbf{G}, \mathbf{D})$ since it is this structure that eventually will yield the most interesting model. In the absence of zero textures, the expression for the rephasing invariants has the particularly simple form $J_{i j}=J_{j i}=\operatorname{Im}\left(m_{i i} m_{j j} m_{i j}^{*} m_{j i}^{*}\right)$ [44]. Taking $i$ and $j$ as defined in eq. (3.5) and writing $J_{i j}=\operatorname{Im}\left(m_{i i} m_{j j}\right) \operatorname{Re}\left(m_{i j}^{2}\right)-\operatorname{Re}\left(m_{i i} m_{j j}\right) \operatorname{Im}\left(m_{i j}^{2}\right)$, it is easy to see that the first condition in eq. (3.5) implies $J_{i j}=0$. Taking instead the indices $i$ and $\bar{k}$, and writing $J_{i \bar{k}}=2 \operatorname{Im}\left(m_{i \bar{k}}^{*} \sqrt{m_{i i} m_{\bar{k} \bar{k}}}\right) \operatorname{Re}\left(m_{i \bar{k}}^{*} \sqrt{m_{i i} m_{\bar{k} \bar{k}}}\right)$, the second condition in eq. (3.5) implies $\sum_{i \neq \bar{k}} \operatorname{Im}\left(m_{i \bar{k}}^{*} \sqrt{m_{i i} m_{\bar{k} \bar{k}}}\right)=0$. From this one can derive the relation $J_{j \bar{k}}=-\frac{\operatorname{Re}\left(m_{i \bar{k}}^{*} \sqrt{m_{i i} m_{\bar{k}}}\right)}{\operatorname{Re}\left(m_{j \bar{k}}^{*} \sqrt{m_{j j} m_{\bar{k} \bar{k}}}\right)} J_{i \bar{k}}$. Therefore there is only one independent, non-zero CP-violating rephasing invariant. This result can be traced back to the fact that with ten fundamental seesaw parameters there is only a single physical $\mathrm{CP}$ violating phase, and implies that although in general for these structures the Dirac phase $\delta$ as well as the two Majorana phases will be non-vanishing, they must all be related.

The second point that we have to address regards the number of different textures that correspond to each structure listed in table 2. It is clear that to be able to confront the various textures with experimental neutrino data, we have to decide an ordering for the lepton fields, to give a precise meaning to the flavour labels $e, \mu, \tau$. Most convenient of course is to fix the ordering in $\hat{m}_{e}$ from light to heavy, so that the entries in the neutrino mixing matrix will keep their usual meaning, and count how many inequivalent textures can be obtained by permuting the rows and columns in $m$. Since $m$ is symmetric, there are in principle six possibilities corresponding to permutations of the indices $1,2,3$. With reference to eq. (3.3), for structure $\mathbf{E}$ we have three ways to choose the index $\bar{k}$ corresponding to the texture zero on the diagonal in $m$ (the first condition). For each of these, there are two ways to chose the index $j$ in the second condition, for a total of six inequivalent textures. For structures $(\mathbf{A}, \mathbf{B}, \mathbf{C}, \mathbf{F})$ there are again three ways to choose the index $\bar{k}$ of the vanishing entry in $m$. However, since the second condition in eq. (3.4) is symmetric under the exchange of the other two indices, $i \leftrightarrow j$, these permutations are equivalent. It is thus sufficient to fix $\bar{k}$ to identify the three inequivalent textures. Structures $(\mathbf{G}, \mathbf{D})$ behave similarly: both conditions in eq. (3.5) are symmetric under the exchange $i \leftrightarrow j$, hence once $\bar{k}$ is fixed, the three textures, which in this case refer to the way the indices $1,2,3$ are assigned to $i, j, \bar{k}$ in eq. (3.5), remain univocally identified. 
As anticipated, in the numerical study we will omit an analysis of the two-zero textures of structure $\mathbf{E}$ since thorough studies already exist in the literature. The three textures belonging to structures $(\mathbf{A}, \mathbf{B}, \mathbf{C}, \mathbf{F})$ have one vanishing entry in $m$, and henceforth will be collectively referred to as textures of "type $\mathbf{1}$ " . The three textures in structures $(\mathbf{G}, \mathbf{D})$ have no zero texture, and will be collectively referred as textures of "type 0". For both types $\mathbf{1}$ and $\mathbf{0}$, a texture can be identified with one of the following three labels

$$
[i j \bar{k}] \in\{[123],[132],[231]\}
$$

where we have chosen for definiteness $i<j$.

\section{Confronting textures with neutrino data}

In this section we study whether any of the neutrino mass textures $m$ belonging to the structures of type $\mathbf{1}$ or $\mathbf{0}$ can be in agreement with the available low-energy neutrino data. These data are encoded in the matrix $m_{\nu}=U^{*} \hat{m}_{\nu} U^{\dagger}$, with $U$ the PMNS matrix which includes the available data on neutrino mixing, while $\hat{m}_{\nu}$ is taken to be in one of the two forms given in eq. (2.8), with $\Delta_{\odot}$ and $\Delta_{\oplus}$ experimentally known and $\mu_{0}$ bounded from above. More explicitly, we need to verify if, given $U$ and $\hat{m}_{\nu}$ (in either NO or IO form), any of the six pairs of relations obtained from eq. (3.4) and eq. (3.5) by index permutations can be satisfied when replacing $m \rightarrow m_{\nu}(\mathrm{NO})$ or $m \rightarrow m_{\nu}(\mathrm{IO})$.

\subsection{Analytical expressions for the Majorana phases}

The neutrino mixing matrix is defined as:

$$
\begin{aligned}
U & =R_{23} U_{13} R_{12} P_{\varphi} \\
& =\left(\begin{array}{ccc}
1 & 0 & 0 \\
0 & c_{23} & s_{23} \\
0 & -s_{23} & c_{23}
\end{array}\right)\left(\begin{array}{ccc}
c_{13} & 0 & s_{12} e^{-i \delta} \\
0 & 1 & 0 \\
-s_{13} e^{i \delta} & 0 & c_{12}
\end{array}\right)\left(\begin{array}{ccc}
c_{12} & s_{12} & 0 \\
-s_{12} & c_{12} & 0 \\
0 & 0 & 1
\end{array}\right)\left(\begin{array}{ccc}
e^{-i \varphi_{1} / 2} & 0 & 0 \\
0 & e^{-i \varphi_{2} / 2} & 0 \\
0 & 0 & 1
\end{array}\right) .
\end{aligned}
$$

where in the second line $c_{i j}=\cos \theta_{i j}$ and $s_{i j}=\sin \theta_{i j}$. The two orthogonal matrices $R_{23}$ and $R_{12}$ and the unitary matrix $U_{13}$ respect the standard parametrization. It is, however, convenient for us to parametrize the Majorana phases as in eq. (4.1), which differs from the more common form $P_{\alpha}=\operatorname{diag}\left(1, e^{i \alpha_{21} / 2}, e^{i \alpha_{31} / 2}\right)$ adopted for example by the Particle Data Group [45]. The two parametrizations are related by $\alpha_{21}=\varphi_{1}-\varphi_{2}$ and $\alpha_{31}=\varphi_{1}$ up to an irrelevant overall phase. After substituting $m \rightarrow m_{\nu}$, each pair of constraints for the two types of textures, eq. (3.4) and eq. (3.5), imply four relations (two for the real and two for the imaginary parts of the equations) between the nine low-energy observables $\theta_{12}, \theta_{13}$, $\theta_{23}, \delta, \varphi_{1}, \varphi_{2}, \mu_{0}, \Delta_{\odot}$ and $\Delta_{\oplus}$. In principle this would allow us to express four parameters in terms of the remaining five, but in practice the conditions are highly non-linear and involve periodic functions, and deriving explicit expressions for the functional dependence of some parameters from the others is not possible.

There is, however, an alternative approach that allows us to solve for the two Majorana phases analytically in terms of $\delta$ and of the mixing angles $\theta_{i j}$. We outline the procedure 
using as an example texture $\mathbf{D}$ in table 2, reproduced here for convenience:

$$
\mathbf{D}: \quad m_{e}^{\dagger} m_{e} \sim\left(\begin{array}{ccc}
\times & \times & 0 \\
\times & \times & 0 \\
0 & 0 & \times
\end{array}\right), \quad \tilde{m} \sim\left(\begin{array}{ccc}
\times & 0 & \times \\
0 & 0 & \times \\
\times & \times & \times
\end{array}\right),
$$

where, as it can be checked explicitly, the entries in $\tilde{m}$ satisfy the relation $\tilde{m}_{11} \tilde{m}_{33}=\tilde{m}_{13}^{2}$. Let us choose a phase convention such that $\left(m_{e}^{\dagger} m_{e}\right)_{12}=\left(m_{e}^{\dagger} m_{e}\right)_{21}^{*}$ is the only complex entry, so that $\tilde{m}$ is real symmetric, and can thus be diagonalised by an orthogonal matrix $O$. Multiplication by a diagonal matrix of phases $P_{\xi}$ is also needed to render all the eigenvalues positive. It is shown in appendix B that, in the chosen phase convention, for matrices of the form of $\tilde{m}$ in eq. (4.2) the eigenvalues with the largest and smallest absolute values have the same sign, and opposite with respect to the sign of the middle eigenvalue. By ordering the absolute values from small to large, without loss of generality (see appendix B) the signature of the eigenvalues can then be taken as $(+,-,+$ ). Therefore $P_{\xi}=\operatorname{diag}(1, i, 1)$. In our basis, the matrix that diagonalizes the neutrino matrix $m$ can then be written as $U_{\xi}=L^{\dagger} O P_{\xi}$, with $L$ the CL mixing matrix. Let us now focus on the third row of $U_{\xi}$, which is unaffected by the matrix $L$ since it is non-trivial only in the upperleft $2 \times 2$ block. Since $O$ has only real entries, we can readily see that $U_{\xi}(3,1)$ and $U_{\xi}(3,3)$ are real, while $U_{\xi}(3,2)$ is purely imaginary. We confront this result with the corresponding third row of the PMNS matrix. Since the phase conventions for $U$ and $U_{\xi}$ are different, to carry out the comparison we need to allow for a relative phase redefinition. This can be done by multiplying $U$ by a generic diagonal matrix of phases $P_{\rho}=\operatorname{diag}\left(e^{i \rho_{1}}, e^{i \rho_{2}}, e^{i \rho_{3}}\right)$. Direct comparison between $\left(P_{\rho} U\right)_{3 j}$ and $\left(U_{\xi}\right)_{3 j}$ for the three elements $j=1,2,3$ yields the following conditions:

$$
\begin{aligned}
& \operatorname{Im}\left(P_{\rho} U\right)_{31}=\operatorname{Im}\left[e^{i\left(\rho_{3}-\frac{\varphi_{1}}{2}\right)}\left(-e^{i \delta} c_{12} c_{23} s_{13}\right)+s_{12} s_{23}\right]=0 \\
& \operatorname{Re}\left(P_{\rho} U\right)_{32}=\operatorname{Re}\left[e^{i\left(\rho_{3}-\frac{\varphi_{2}}{2}\right)}\left(-e^{i \delta} s_{12} c_{23} s_{13}\right)-c_{12} s_{23}\right]=0 \\
& \operatorname{Im}\left(P_{\rho} U\right)_{33}=\operatorname{Im}\left[e^{i \rho_{3}} c_{13} c_{23}\right]=0 .
\end{aligned}
$$

The last equation fixes $\rho_{3}=0$ or $\pi$, and we can then obtain $\varphi_{1}$ and $\varphi_{2}$ from the other two equations in terms of $\delta$ and $\theta_{i j}$. The same procedure can be carried out for all the other textures. The final result depends on the particular texture $[i j \bar{k}]$ since different rows of the PMNS matrix $U$ are selected for the comparison for the different cases, but it does not depend on whether the texture is of type $\mathbf{1}$ or $\mathbf{0}$. We obtain:

$$
\tan \left(\frac{\varphi_{1}}{2}\right)=\frac{\tan \delta}{\Omega_{[i j \bar{k}]}^{\left(\varphi_{1}\right)}}, \quad \tan \left(\frac{\varphi_{2}}{2}\right)=-\frac{\Omega_{[i j \bar{k}]}^{\left(\varphi_{2}\right)}}{\tan \delta},
$$

with

$$
\begin{aligned}
\Omega_{[123]}^{\left(\varphi_{1}\right)} & =1-\frac{t_{12} t_{23}}{s_{13} \cos \delta}, & \Omega_{[123]}^{\left(\varphi_{2}\right)} & =1+\frac{t_{23}}{t_{12} s_{13} \cos \delta}, \\
\Omega_{[132]}^{\left(\varphi_{1}\right)} & =1+\frac{t_{12}}{s_{13} t_{23} \cos \delta}, & \Omega_{[132]}^{\left(\varphi_{2}\right)} & =1-\frac{1}{t_{12} s_{13} t_{23} \cos \delta}, \\
\Omega_{[231]}^{\left(\varphi_{1}\right)} & =1, & \Omega_{[231]}^{\left(\varphi_{2}\right)} & =1,
\end{aligned}
$$


where $t_{i j}=\tan \theta_{i j}$ and $s_{i j}=\sin \theta_{i j}$. Note that for the texture [231] the phase relations are particularly simple: $\varphi_{1}=\varphi_{2}-\pi=2 \delta$.

\subsection{Numerical analysis}

The possibility of writing down analytic relations for the Majorana phases in terms of the other low-energy parameters is a nice result, but given that $\varphi_{1,2}$ are the most difficult quantities to determine experimentally, in practice there is little hope to directly test the model via eq. (4.4). Still, it is useful to eliminate $\varphi_{1,2}$ from eq. (3.4) and eq. (3.5) by expressing them in terms of the other parameters. Note, however, that in doing so we implicitly reduce the number of constraints, since the existence of matrix textures that yields the relations eq. (3.4) and eq. (3.5) is also what has been used to determine the functional dependence of $\varphi_{1,2}$ on $\delta$ and $\theta_{i j}$. In short, although the number of independent parameters has now dropped from nine to seven, the number of independent constraints represented by each pair of complex conditions has also (implicitly) dropped from four to two. Thus only two additional quantities can be determined in terms of the others five by solving for the two real and two imaginary conditions for each one of the two cases. As we have already mentioned, it is not possible to analytically extract from eq. (3.4) and eq. (3.5) the dependence of any pair of parameters from the others, and thus we need to resort to numerical methods.

We proceed as follows: respectively for textures of type $\mathbf{1}$ and $\mathbf{0}$, we combine the pair of conditions in a single expression:

$$
\begin{aligned}
& \mathcal{C}_{\mathbf{1}}=\eta^{-4}\left|m_{i i} m_{j j}-m_{i j}^{2}\right|^{2}+\eta^{-2}\left|m_{\bar{k} \bar{k}}\right|^{2} \\
& \mathcal{C}_{\mathbf{0}}=\eta^{-4}\left|m_{i i} m_{j j}-m_{i j}^{2}\right|^{2}+\eta^{-4}\left|\sum_{\ell \neq \bar{k}}\left(m_{\ell \bar{k}}^{*} \sqrt{m_{\ell \ell} m_{\bar{k} \bar{k}}}-\left|m_{\ell \ell} m_{\bar{k} \bar{k}}\right|\right)\right|^{2},
\end{aligned}
$$

where

$$
\eta^{2}=\operatorname{tr}\left(m^{\dagger} m\right)=\sum_{i} \mu_{i}^{2}
$$

is a normalisation factor that ensure that $\mathcal{C}_{\mathbf{1 , 0}}$ are dimensionless and, not to clutter the notations, we have omitted the label $\nu$ from the matrix elements: $\left(m_{\nu}\right)_{i j}=m_{i j}$. Each matrix element $m_{i j}=m_{i j}(\{p\})$ depends on the seven parameters $\{p\}=\left\{\theta_{12}, \theta_{13}, \theta_{23}, \delta, \mu_{0}, \Delta_{\odot}, \Delta_{\oplus}\right\}$, and so do the two functions $\mathcal{C}_{\mathbf{1 , 0}}=\mathcal{C}_{\mathbf{1 , 0}}(\{p\})$. We have to search for values of these parameters that yield $\mathcal{C}_{\mathbf{1 , 0}}=0$. Since $\mathcal{C}_{\mathbf{1 , 0}, 0}$ depend only on the absolute values of the conditions eqs. (3.4) $-(3.5), \mathcal{C}_{\mathbf{1}, \mathbf{0}}=0$ necessarily corresponds to a global minimum, and thus the search can be carried out via numerical minimization. Let us note at this point that $\delta \rightarrow-\delta$ corresponds to $U \rightarrow U^{*}$ which in turn implies $m_{i j} \rightarrow m_{i j}^{*}$. However, under complex conjugation all the conditions in eqs. (3.4)-(3.5) remain invariant, so that we cannot expect to obtain information about the half-plane in which $\delta$ lies. In carrying out the minimisation, it is convenient to restrict the parameter space $\{p\}$ by excluding regions that are experimentally known to be highly unlikely. This can be done, for each numerical run, by sampling the values of the most precisely measured quantities from normal distributions with mean and variance corresponding to their experimental values. In principle we could consider $\Delta_{\odot}, \Delta_{\oplus}, \theta_{12}, \theta_{13}$ and $\theta_{23}$ as measured, and derive from minimization the values 


\begin{tabular}{|cccccccc|}
\hline $\begin{array}{c}\text { No. of zero } \\
\text { textures }\end{array}$ & \multicolumn{3}{c}{ Normal Ordering } & & \multicolumn{3}{c|}{ Inverted Ordering } \\
\cline { 2 - 4 } \cline { 6 - 8 } & {$[123]$} & {$[132]$} & {$[231]$} & & {$[123]$} & {$[132]$} & {$[231]$} \\
\hline $\mathbf{1}$ & $\boldsymbol{X}$ & $\boldsymbol{x}$ & $\boldsymbol{X}$ & & $\boldsymbol{x}$ & $\boldsymbol{x}$ & $\boldsymbol{X}$ \\
$\mathbf{0}$ & $\boldsymbol{X}$ & $\boldsymbol{V}$ & $\boldsymbol{X}$ & & $\boldsymbol{X}$ & $\boldsymbol{X}$ & $\boldsymbol{X}$ \\
\hline
\end{tabular}

Table 3. Summary of the numerical results. A texture labeled with a large x-mark $(\boldsymbol{X})$ indicates that the conditions $\mathcal{C}_{\mathbf{1}, \mathbf{0}}=0$ cannot be satisfied. A small x-mark $(\boldsymbol{x})$ indicates that $\mathcal{C}_{\mathbf{1 , 0}}=0$ can only be obtained for values of $\theta_{23}$ or $\mu_{0}$ that are experimentally excluded. The checkmark $\boldsymbol{V}$ for the texture [132] with no texture zero highlights the only viable possibility.

of the neutrino mass scale $\mu_{0}$ and of $\delta$. However, global fits to neutrino data indicate that the shape of $\chi^{2}$ for $\theta_{23}$ is far from parabolic, resembling rather a bimodal distribution, with two different minima respectively located in the first and second octant (see e.g. [26]), so that using a normal distribution for this parameter is not justified. We have then opted for minimizing with respect to the three variables $\mu_{0}, \delta$ and $\theta_{23}$, since in this way we can also put in evidence their reciprocal functional dependence.

\subsection{Results}

The complete numerical analysis requires twelve numerical minimizations corresponding to three textures $[i j \bar{k}]$ for each one of the two structures $\mathbf{1}$ and $\mathbf{0}$, that have to be confronted with neutrino data assuming in turn NO or IO. For each case there are three possible outcomes:

1. There is no set of values for $\mu_{0}, \delta$ and $\theta_{23}$ that can yield $\mathcal{C}=0$. These cases are thus inconsistent with the constraints and can be immediately discarded. The seven possibilities marked with a large $\mathrm{x}$-mark $(\boldsymbol{X})$ in table 3 belong to this class. This includes all models with $[i j \bar{k}]=[231]$.

2. There exists a region where $\mathcal{C}=0$, but the required values of the parameters are incompatible with experimental limits. Hence also these possibilities are ruled out. Four cases belong to this class, and are marked in the table with a small x-mark $(\boldsymbol{x})$. They correspond to the two texture [123] and [132] for structure $\mathbf{1}$. When confronted with NO, $\mathcal{C}_{\mathbf{1}}=0$ requires $\theta_{23} \sim \frac{2 \pi}{5}$ for [123] and $\theta_{23} \sim \frac{\pi}{10}$ for [132]. When confronted with IO, for both textures $\mu_{0} \gg 1 \mathrm{eV}$ is required to satisfy the condition.

3. If $\mathcal{C}=0$ is instead satisfied for values of the parameters within the experimental limits, the texture is viable. We find only one viable case, that is highlighted in the table with a check-mark $(\boldsymbol{V})$. It corresponds to texture [132] for a neutrino mass matrix structure without texture zeros, that agrees well with all neutrino data when NO is assumed. We label this case $[132]_{\mathbf{0}}$.

Besides being viable, texture $[132]_{0}$ also yields rather precise predictions, which are depicted in figures 1 and 2. Let us explain how these plots are obtained and their meaning. For a given set of reference values for $\Delta_{\odot}, \Delta_{\oplus}, \theta_{12}, \theta_{13}$, the equation $\mathcal{C}_{\mathbf{0}}=0$ represents a line in the $\left(\delta, \mu_{0}, \theta_{23}\right) 3 \mathrm{D}$ space. When the reference values are allowed to vary within 

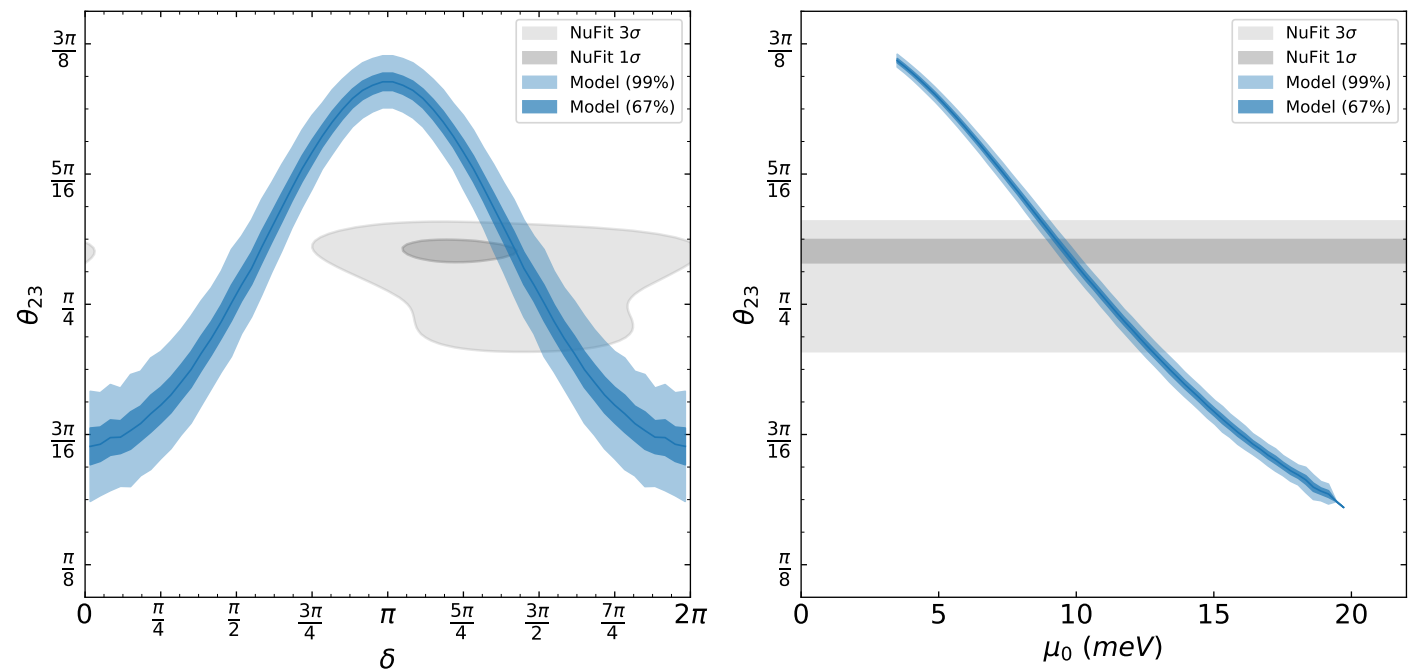

Figure 1. Left panel: functional dependence of $\theta_{23}$ vs. $\delta$ at the $\mathcal{C}_{\mathbf{0}}=0$ absolute minimum. The dark (light) gray region depicts the $1 \sigma(3 \sigma)$ experimentally allowed limits from ref. [26]. The meaning of the dark and light blue regions is explained in the text. Right panel: same for $\theta_{23}$ vs. $\mu_{0}$.

their experimental errors, this line is replaced by a volume. We construct this volume by generating a large set of $\mathcal{C}_{\mathbf{0}}=0$ points for which the reference values are drawn from the experimental distributions for the corresponding parameters, assumed to be normal and uncorrelated. The points are then binned according to their value of $\delta$, and for each bin the mean $\mu$ and standard deviation $\sigma$ for the values of $\theta_{23}$ and $\mu_{0}$ within that bin are computed. We then draw two volumes, corresponding respectively to $\mu \pm \sigma$ (dark blue) and $\mu \pm 3 \sigma$ (light blue). Figure 1 depicts the projection of these volumes on the two planes $\left(\delta, \theta_{23}\right)$ (left panel) and $\left(\mu_{0}, \theta_{23}\right)$ (right panel), that is the marginalisation of the functional dependences $\theta_{23}=\theta_{23}(\delta)$ and $\theta_{23}=\theta_{23}\left(\mu_{0}\right)$ respectively over $\mu_{0}$ and $\delta$. Of course these regions should not be interpreted as representing an experimental statistical significance, but they still give a qualitatively meaningful account of the accuracy with which the functional dependences can be determined. For example, from the plot on the right we see that $\theta_{23}$ and $\mu_{0}$ are approximately linearly anticorrelated, and that the spread around the central line is rather small. An interesting prediction that can be read out from the left plot is that approximate maximal atmospheric mixing $\theta_{23} \approx \frac{\pi}{4}$ favours nearly maximal $\mathrm{CP}$ violation $\delta \approx \frac{\pi}{2}, \frac{3 \pi}{2}$. In both plots the dark and the light grey regions depict respectively the $1 \sigma$ and $3 \sigma$ experimental uncertainties on $\theta_{23}$ and $\delta$, taken from ref. [26].

In figure 2 we plot the functional dependence between $\mu_{0}$ and $\delta$ at the absolute minimum $\mathcal{C}_{\mathbf{0}}=0$. The gray regions correspond to the $1 \sigma$ (dark) and $(3 \sigma)$ (light) experimentally allowed limits for $\delta$ from ref. [26]. The dark (light) green box encloses the values of $\delta$ and $\mu_{0}$ for which the corresponding values of $\theta_{23}$ in the two plots in figure 1 remain within the $1 \sigma$ $(3 \sigma)$ experimental limits. The vertical sides of the boxes are located at $\delta=4.45_{-0.24(0.32)}^{+0.35(1.06)}$ rad. We see that the prediction of the model for $\delta$ in terms of the experimentally allowed values of $\theta_{23}$ is in good agreement with the direct determination from the global fit in ref. [26]. The horizontal sides are located at $\mu_{0}=9.5_{-0.5(1.0)}^{+0.7(3.6)} \mathrm{meV}$, and provide a rather 


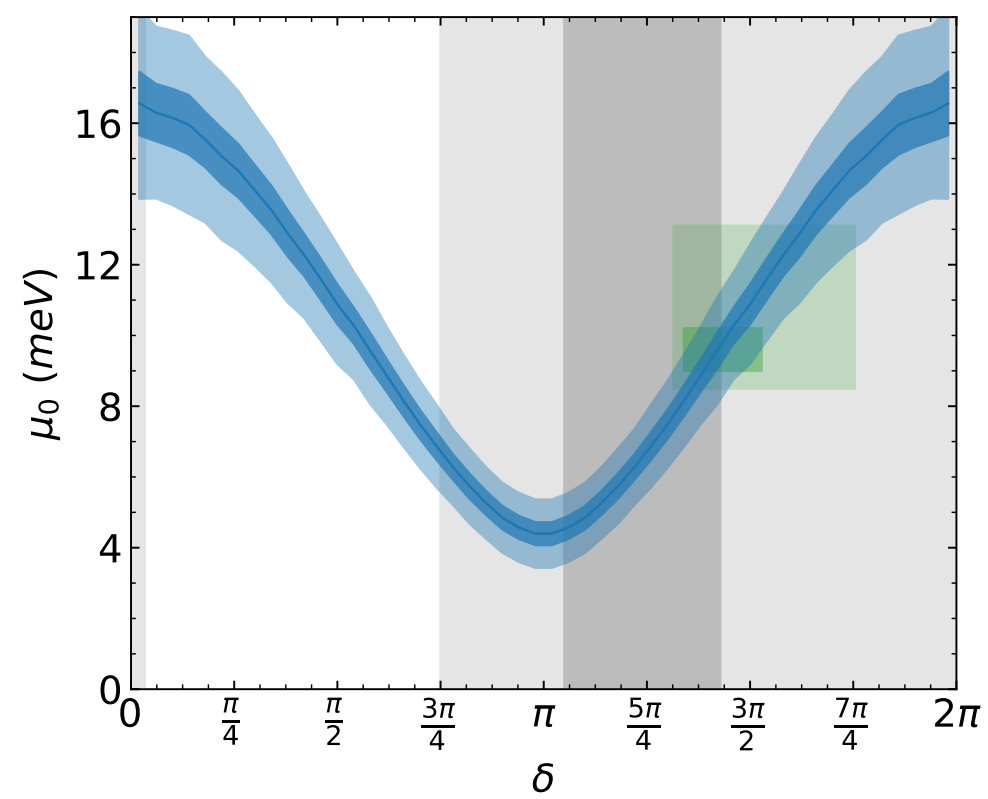

Figure 2. Functional dependence of $\mu_{0}$ vs. $\delta$ at the $\mathcal{C}_{\mathbf{0}}=0$ absolute minimum. The dark (light) gray area corresponds to the $1 \sigma(3 \sigma)$ experimentally allowed region for $\delta$ from ref. [26]. The dark (light) green area encloses the values of $\delta$ and $\mu_{0}$ for which the corresponding values of $\theta_{23}$ (see the two plots in figure 1) remain within the $1 \sigma(3 \sigma)$ experimental limits.

precise prediction for the neutrino mass scale. There is in principle a second set of green boxes that can be obtained by reflection with respect to the $\delta=\pi$ vertical line. This is because, as mentioned above, the texture conditions do not distinguish between values of $\delta$ in the first or in the second half plane. However, values of $\delta$ centred around $\frac{\pi}{2}$ are disfavoured by direct measurements, so that we have not drawn the corresponding boxes.

\section{Concluding remarks}

The quest for a structure of the neutrino mass matrix that can agree with all the available neutrino data and at the same time yield testable predictions, has represented a major effort pursued in the last two decades by many theorists. Predictivity is obviously linked to a reduction in the number of free parameters, and a simple way to achieve this is to assume that some entries in the neutrino mass matrix vanish. For this reason neutrino mass matrix structures with one or two vanishing entries have been thoroughly studied in the literature. In this paper we have adopted a top-down approach. We have assumed that the neutrino mass matrix is described by an effective Weinberg operator that arises from the type-I seesaw, and that a U(1) flavour symmetry exists which determines the structure of the renormalizable high-energy Lagrangian. As a first step we have searched for $\mathrm{U}(1)$ symmetries able to reduce the number of seesaw parameters to the minimum number required to account qualitatively for three non-zero neutrino masses and mixing angles while allowing for $\mathrm{CP}$ violation in the lepton sector. We have given a complete 
classification of the various possibilities that can arise when the number of leptonic Higgs scalars carrying different $\mathrm{U}(1)$ charges is restricted to two. We have then identified the corresponding mass matrix structures, as well as the different textures that can arise in the low-energy effective theory. We have found that in some cases mass matrices with one or two zero textures do arise, corresponding to the condition $m_{i j}=0$ for some values of the indices. However, more generally these symmetries can produce complicated conditions involving several entries of the mass matrix at the same time, that can be written as $f\left(m_{i j}\right)=0$ with $f$ some well-defined function. These conditions have the same effect of zero-textures in reducing the number of parameters, as they allow one to express (although only implicitly) some parameters in terms of the others. We have then extended the meaning of the word 'texture' to refer also to the possibility of exact relations between the parameters. We have found that some cases with two-zero textures can indeed be generated by a U(1) symmetry, while others that have also been considered in the literature cannot, at least in the simplest case of two leptonic Higgs doublets. Only one of the matrices with two-zero textures we have found is viable, as it has been confirmed by recent up-to-date numerical analysis $[32,33]$. Textures with a single vanishing entry are also generated, but none of them are phenomenologically viable.

Finally, we have identified a set of six mass matrices with no vanishing entries, and we have singled out a unique case that, for normal ordering, is in excellent agreement with all available neutrino data, and predicts $\mu_{0} \sim 10 \mathrm{meV}$ for the lightest neutrino mass, $\delta \sim \frac{3 \pi}{2}$ for the $\mathrm{CP}$ violating Dirac phase, and it also determines the two Majorana phases via the analytic expressions in the second line of eq. (4.5) (thus the rate of $0 \nu 2 \beta$ decay can also be predicted, but it remains well below the sensitivity of present and foreseeable future experiments). We stress that for mass matrices of this type it would be impossible to identify the underlying U(1) symmetry, even in the limit of infinite experimental precision in the determination of all the low-energy parameters. This not only because the symmetry leaves no obvious trace in the structure of the neutrino mass matrix, but even more importantly because different symmetries (in the sense of different charge assignments for the fields that yield different high-energy structures) can give rise to the same low-energy structure. For example, the successful no-zero texture that we have identified as viable and predictive, can be generated by the two different high-energy structures that we have labeled as G: 2(2)[12][13] and D: 1(3)[]\{13\}. It is for this reason that it is appropriate to define this type of symmetries as covert. Additional information that could help discriminating among the different symmetries might come from the study of leptogenesis, since this could give some insight into the structure of the fundamental high-energy mass matrices. For example, in G: 2(2)[12][13] the structure of $M_{N}$ implies two degenerate states with opposite CP transformations which correspond to one heavy Dirac neutrino, and this strongly disfavours the possibility that leptogenesis could be realised in this scenario, while for $\mathbf{D}: 1(3)[]\{13\}$ there is no apparent reason why leptogenesis should not be successful. A study of the high-energy signatures that could distinguish between $\mathbf{G}$ and $\mathbf{D}$ is, however, beyond the scope of the present paper.

One should also keep in mind that a fundamental ingredient of our construction is that in general the lepton fields all carry different U(1) charges. Since U(1) is spontaneously 
broken, there is a Nambu-Goldstone boson (NGB) $a(x)$ that couples non-diagonally to the lepton fields, so that lepton flavour violating (LFV) processes like $\mu \rightarrow e a, \tau \rightarrow \mu a$ etc. will unavoidably occur. However, since all the couplings of this NGB are suppressed as $1 / f_{\phi}$, if the value of this scale is sufficiently large, say $f_{\phi} \gg 10^{9} \mathrm{GeV}$, the rate of all these processes can remain safely below the experimental bounds.

There are other two issues that deserve some comments. After $\mathrm{U}(1)$ is broken, the vanishing entries in the high energy matrices can get lifted by corrections, and it is important to see if their size can be kept sufficiently small so that the $f\left(m_{i j}\right) \simeq 0$ conditions remain satisfied to a sufficiently good level of approximation. The second issue is related to the presence of two Higgs doublets coupled to the lepton fields, that can give rise to LFV processes that must also remain under control. Both these issues depend on the details of the scalar sector. After $\mathrm{U}(1)$ breaking the leading corrections to the texture zero will involve the Higgs mixing term originating from the couplings in eq. (2.1). The relevant parameter in case 1 is $m_{12}^{2}=\mu f_{\phi}$ with $\mu$ a coupling with dimension of a mass, and in case 2 is $m_{12}^{2}=y f_{\phi}^{2}$ with $y$ some dimensionless coupling. To discuss one example, let us consider the textures of case $\mathbf{G}$, see eq. (A.4). Since the Yukawa entry in the Dirac matrix $\left(m_{D}\right)_{1,2}$ is non vanishing, from the couplings $\bar{N}_{1} \ell_{2} H_{1}$ and $\bar{N}_{2} \ell_{2} H_{2}$ one can draw a mixed self-energy loop diagram connecting $N_{1}$ and $N_{2}$. This requires an insertion of the U(1) breaking spurion $m_{12}^{2}$ on the scalar field line and, recalling that two non-vanishing entries in the same row or column in $m_{D}$ must necessarily involve different Higgs doublets, this is indeed a general requirement. Therefore, besides the suppression from loop factors, it would be also desirable to keep the size of $m_{12}^{2}$ as small as possible. On the other hand, keeping under control Higgs mediated LFV interactions requires that below some sufficiently large scale one heavy Higgs with mass $m_{h}$ will decouple, leaving only one light Higgs with mass $m_{l} \sim 125 \mathrm{GeV}$ and with the same properties of the SM Higgs. The two issues can be simultaneously solved for example by taking in the scalar potential a large (positive) mass square parameter $m_{2}^{2} \sim m_{h}^{2} \gg v^{2}$ for, say, $H_{2}$, and a relatively smaller mixing term, e.g. $m_{12}^{2} / m_{h}^{2} \sim 10^{-3}$. With $m_{2} \sim 100 \mathrm{TeV}$ all LFV effects remain well under control, and the size of the mixed $N_{1}-N_{2}$ self-energy diagrams, which is of order $\sim M_{N} \frac{Y_{D}^{2}}{16 \pi^{2}} \frac{m_{12}^{2}}{m_{h}^{2}}$ with $Y_{D}$ a Yukawa coupling, gets an additional strong suppression $\sim 10^{-3}$. A scalar potential with these features might even help to account for the lightness of the leptons coupled to $\mathrm{H}_{2}$, since their couplings to the light Higgs that acquires a vev is suppressed by the mixing.

Finally, it could be tempting to interpret the U(1) symmetry as a Peccei-Quinn (PQ) symmetry, and $a(x)$ as the axion. In order to do so it is sufficient to extend the symmetry to the quark sector. If the requirement of enforcing minimality in the number of parameters is maintained also for the quarks, then all the viable possibilities have already been classified in ref. [24]. In all cases the U(1) symmetry has a QCD anomaly and thus it is a PQ symmetry. In an extended model of this type, the high-energy scale $f_{\phi}$ would then acquire an even more fundamental role, since a value $f_{\phi} \sim 10^{10}-10^{12} \mathrm{GeV}$ would at the same time be a natural one for the type-I seesaw, optimal for successful leptogenesis, safe with respect to FCNC and LFV decays, and preferred by the vast majority of axion models. 


\section{Acknowledgments}

F.B. and E.N. are supported by the INFN "Iniziativa Specifica" Theoretical Astroparticle Physics (TAsP-LNF). The work of L.D.L. is supported by the Marie Skłodowska-Curie Individual Fellowship grant AXIONRUSH (GA 840791) and the ERC grant NEO-NAT. F.M. is supported by MINECO grant FPA2016-76005-C2-1-P, by Maria de Maetzu program grant MDM-2014-0367 of ICCUB and 2017 SGR 929. F.M. acknowledges the INFN Laboratori Nazionali di Frascati for hospitality and financial support.

\section{A U(1) charges for viable neutrino mass textures}

We list in this appendix the values of the $\mathrm{U}(1)$ charges that yield the two viable textures for the neutrino mass matrix. The first possibility corresponds to a structure of type $\mathbf{E}$, and to the specific texture in which the neutrino mass matrix $m$ has two vanishing entries in the $(1,1)$ and $(1,3)$ positions:

$$
\mathbf{E}: \quad m=\left(\begin{array}{ccc}
0 & \times & 0 \\
\times & \times & \times \\
0 & \times & \times
\end{array}\right) .
$$

There is a unique form of the matrices $M_{N}, m_{D}$ and $m_{e}$ which yields this low-energy texture which, according to the notation introduced in section 3.1, is labeled as 2(3)[13][]:

$$
\mathbf{E}: \quad M_{N}=\left(\begin{array}{ccc}
0 & \times & \times \\
\times & 0 & 0 \\
\times & 0 & \times
\end{array}\right), \quad m_{D}=\left(\begin{array}{ccc}
\times & 0 & \times \\
0 & \times & 0 \\
0 & 0 & \times
\end{array}\right), \quad m_{e}=\left(\begin{array}{ccc}
\times & 0 & 0 \\
0 & \times & 0 \\
0 & 0 & \times
\end{array}\right) .
$$

Modulo an overall change of sign, the set of $U(1)$ charges for this case is also unique:

$$
\mathbf{E}: \quad \mathcal{X}(N)=(-3,1,5), \quad \mathcal{X}(\ell)=(-5,-1,7), \quad \mathcal{X}(e)=(-7,1,5),
$$

Numerical confrontations with up to date neutrino data have been recently carried out in refs. [32, 33], and confirm that the two-zero texture mass matrix in eq. (A.1) is viable and predictive. The other viable and predictive possibility corresponds to a mass matrix with no zero textures, but with the entries constrained by the conditions in eq. (3.5) with $[i j \bar{k}]=[132]$. Such a texture can be generated by two different high-energy structures. One is of type $\mathbf{G}$ with label 2(2)[12][13], as given in table 2. The mass matrices are:

$$
\text { G : } \quad M_{N}=\left(\begin{array}{ccc}
\times & 0 & 0 \\
0 & 0 & \times \\
0 & \times & 0
\end{array}\right), \quad m_{D}=\left(\begin{array}{ccc}
\times & \times & 0 \\
0 & \times & 0 \\
0 & 0 & \times
\end{array}\right), \quad m_{e}=\left(\begin{array}{ccc}
\times & 0 & \times \\
0 & \times & 0 \\
0 & 0 & \times
\end{array}\right),
$$

which result from a unique set of charges:

$$
\mathbf{G}: \quad \mathcal{X}(N)=(1,5,-7), \quad \mathcal{X}(\ell)=(-1,3,-5), \quad \mathcal{X}(e)=(-3,5-7) .
$$

The other is a structure of type $\mathbf{D}$ with texture $1(3)[]\{13\}$, that is a permutation of the case listed in table 2, and for which the mass matrices are:

$$
\text { D : } \quad M_{N}=\left(\begin{array}{ccc}
\times & 0 & \times \\
0 & 0 & \times \\
\times & \times & 0
\end{array}\right), \quad m_{D}=\left(\begin{array}{ccc}
\times & 0 & 0 \\
0 & \times & 0 \\
0 & 0 & \times
\end{array}\right), \quad m_{e}=\left(\begin{array}{ccc}
\times & 0 & \times \\
0 & \times & 0 \\
0 & 0 & \times
\end{array}\right) \text { or }\left(\begin{array}{ccc}
\times & 0 & 0 \\
0 & \times & 0 \\
\times & 0 & \times
\end{array}\right) \text {. }
$$


The set of charges that enforce these textures can be written as:

$$
\mathbf{D}: \quad \mathcal{X}(N)=(1,5,-3), \quad \mathcal{X}(\ell)=(0, p,-2), \quad \mathcal{X}(e)=(q, p \pm 1, q-2),
$$

with $p=5 \pm 1$ and $q= \pm 1$. The structure of these matrices is invariant with respect to a particular choice for $p$, while the different values of $q$ simply give $\left(m_{e}\right)_{13} \neq 0$ or $\left(m_{e}\right)_{31} \neq 0$.

\section{B Signature of the eigenvalues for texture D}

The structure of the neutrino mass matrix $\tilde{m}$ for texture $\mathbf{D}$ in the basis in which the CL charges are well defined is:

$$
\tilde{m}=\left(\begin{array}{ccc}
m_{11} & 0 & \sqrt{m_{11} m_{33}} \\
0 & 0 & m_{23} \\
\sqrt{m_{11} m_{33}} & m_{23} & m_{33}
\end{array}\right)
$$

We want to prove that, in the basis in which all the entries in $\tilde{m}$ are real, when ordered according to increasing absolute values, the sign of the second eigenvalue $\mu_{2}$ is discordant from the signs of the eigenvalues with the smallest and largest absolute values $\mu_{1}$ and $\mu_{3}$. Let us write down the coefficients of the characteristic equation $\operatorname{det}(\tilde{m}-\mu I)=0$ :

$$
\begin{aligned}
\operatorname{tr}(\tilde{m}) & =m_{11}+m_{33}=\mu_{1}+\mu_{2}+\mu_{3}, \\
c_{1}(\tilde{m}) & =m_{23}^{2}=-\left(\mu_{1} \mu_{2}+\mu_{1} \mu_{3}+\mu_{2} \mu_{3}\right), \\
\operatorname{det}(\tilde{m}) & =-m_{11} m_{23}^{2}=\mu_{1} \mu_{2} \mu_{3},
\end{aligned}
$$

where $c_{1}$ is the coefficient of the linear term. From this we can derive a useful relation:

$$
R=-\left[c_{1}(\tilde{m}) \operatorname{tr}(\tilde{m})+\operatorname{det}(\tilde{m})\right]=-m_{33} m_{23}^{2}=\left(\mu_{1}+\mu_{2}\right)\left(\mu_{1}+\mu_{3}\right)\left(\mu_{2}+\mu_{3}\right) .
$$

Now the signs of $m_{11}$ and $m_{33}$ must be concordant to ensure that $m_{13}$ is real. If $m_{11}, m_{33}>$ 0 the determinant is negative and we have either three negative eigenvalues or one negative and two positive. Since $\operatorname{tr}(\tilde{m})>0$ the first possibility is excluded. Next we have to ensure that $R<0$. It is readily seen that the ordered signatures $(-,+,+)$ and $(+,+,-)$ for the eigenvalues give $R>0$ and are excluded, while $(+,-,+)$ ensures the correct result. If $m_{11}, m_{33}<0$ the same reasoning gives the signature $(-,+,-)$, hence the sign of $\mu_{2}$ is always discordant from that of $\mu_{1}$ and $\mu_{3}$. The two cases $m_{11}, m_{33}>0$ and $m_{11}, m_{33}<0$ are related by a trivial rephasing of all the lepton fields by $e^{i \pi / 2}$. Therefore assuming the eigenvalues signature $(+,-,+)$ is without loss of generality.

Open Access. This article is distributed under the terms of the Creative Commons Attribution License (CC-BY 4.0), which permits any use, distribution and reproduction in any medium, provided the original author(s) and source are credited. 


\section{References}

[1] C.D. Froggatt and H.B. Nielsen, Hierarchy of quark masses, Cabibbo angles and CP-violation, Nucl. Phys. B 147 (1979) 277 [INSPIRE].

[2] M. Leurer, Y. Nir and N. Seiberg, Mass matrix models, Nucl. Phys. B 398 (1993) 319 [hep-ph/9212278] [INSPIRE].

[3] M. Leurer, Y. Nir and N. Seiberg, Mass matrix models: the sequel, Nucl. Phys. B 420 (1994) 468 [hep-ph/9310320] [INSPIRE].

[4] M. Dine, R.G. Leigh and A. Kagan, Flavor symmetries and the problem of squark degeneracy, Phys. Rev. D 48 (1993) 4269 [hep-ph/9304299] [InSPIRE].

[5] L.E. Ibáñez and G.G. Ross, Fermion masses and mixing angles from gauge symmetries, Phys. Lett. B 332 (1994) 100 [hep-ph/9403338] [InSPIRE].

[6] T. Banks, Y. Grossman, E. Nardi and Y. Nir, Supersymmetry without R-parity and without lepton number, Phys. Rev. D 52 (1995) 5319 [hep-ph/9505248] [INSPIRE].

[7] E. Dudas, C. Grojean, S. Pokorski and C.A. Savoy, Abelian flavor symmetries in supersymmetric models, Nucl. Phys. B 481 (1996) 85 [hep-ph/9606383] [INSPIRE].

[8] N. Irges, S. Lavignac and P. Ramond, Predictions from an anomalous U(1) model of Yukawa hierarchies, Phys. Rev. D 58 (1998) 035003 [hep-ph/9802334] [INSPIRE].

[9] J.M. Mira, E. Nardi and D.A. Restrepo, Nonanomalous horizontal U(1) $)_{H}$ gauge model of flavor, Phys. Rev. D 62 (2000) 016002 [hep-ph/9911212] [INSPIRE].

[10] J.K. Elwood, N. Irges and P. Ramond, Family symmetry and neutrino mixing, Phys. Rev. Lett. 81 (1998) 5064 [hep-ph/9807228] [INSPIRE].

[11] A. Pomarol and D. Tommasini, Horizontal symmetries for the supersymmetric flavor problem, Nucl. Phys. B 466 (1996) 3 [hep-ph/9507462] [InSPIRE].

[12] R. Barbieri, G.R. Dvali and L.J. Hall, Predictions from a U(2) flavor symmetry in supersymmetric theories, Phys. Lett. B 377 (1996) 76 [hep-ph/9512388] [INSPIRE].

[13] R. Barbieri, L.J. Hall, S. Raby and A. Romanino, Unified theories with U(2) flavor symmetry, Nucl. Phys. B 493 (1997) 3 [hep-ph/9610449] [INSPIRE].

[14] R. Barbieri, L.J. Hall and A. Romanino, Consequences of a U(2) flavor symmetry, Phys. Lett. B 401 (1997) 47 [hep-ph/9702315] [INSPIRE].

[15] C.D. Carone and L.J. Hall, Neutrino physics from a U(2) flavor symmetry, Phys. Rev. D 56 (1997) 4198 [hep-ph/9702430] [INSPIRE].

[16] E. Nardi, Naturally large Yukawa hierarchies, Phys. Rev. D 84 (2011) 036008 [arXiv:1105.1770] [INSPIRE].

[17] R. Alonso, M.B. Gavela, L. Merlo and S. Rigolin, On the scalar potential of minimal flavour violation, JHEP 07 (2011) 012 [arXiv: 1103.2915] [INSPIRE].

[18] J.R. Espinosa, C.S. Fong and E. Nardi, Yukawa hierarchies from spontaneous breaking of the $\mathrm{SU}(3)_{L} \times \mathrm{SU}(3)_{R}$ flavour symmetry?, JHEP 02 (2013) 137 [arXiv: 1211.6428] [INSPIRE].

[19] C.S. Fong and E. Nardi, Quark masses, mixings and CP-violation from spontaneous breaking of flavor SU $(3)^{3}$, Phys. Rev. D 89 (2014) 036008 [arXiv:1307.4412] [INSPIRE]. 
[20] L.F. Duque, D.A. Gutierrez, E. Nardi and J. Norena, Fermion mass hierarchy and non-hierarchical mass ratios in $\mathrm{SU}(5) \times \mathrm{U}(1)_{F}$, Phys. Rev. D 78 (2008) 035003 [arXiv:0804.2865] [INSPIRE].

[21] F. Wang and Y.-X. Li, Generalized Froggatt-Nielsen mechanism, Eur. Phys. J. C 71 (2011) 1803 [arXiv: 1103.6017] [inSPIRE].

[22] E. Nardi, D. Restrepo and M. Velasquez, Neutrino masses in $\mathrm{SU}(5) \times \mathrm{U}(1)_{F}$ with adjoint flavons, Eur. Phys. J. C 72 (2012) 1941 [arXiv:1108.0722] [InSPIRE].

[23] Y. Reyimuaji and A. Romanino, Can an unbroken flavour symmetry provide an approximate description of lepton masses and mixing?, JHEP 03 (2018) 067 [arXiv:1801.10530] [INSPIRE].

[24] F. Björkeroth, L. Di Luzio, F. Mescia and E. Nardi, U(1) flavour symmetries as Peccei-Quinn symmetries, JHEP 02 (2019) 133 [arXiv:1811.09637] [INSPIRE].

[25] E. Ma, Pathways to naturally small neutrino masses, Phys. Rev. Lett. 81 (1998) 1171 [hep-ph/9805219] [INSPIRE].

[26] I. Esteban et al., Global analysis of three-flavour neutrino oscillations: synergies and tensions in the determination of $\theta_{23}, \delta_{\mathrm{CP}}$ and the mass ordering, JHEP 01 (2019) 106 [arXiv: 1811.05487] [INSPIRE].

[27] P.F. de Salas et al., Status of neutrino oscillations 2018: $3 \sigma$ hint for normal mass ordering and improved CP sensitivity, Phys. Lett. B 782 (2018) 633 [arXiv:1708.01186] [INSPIRE].

[28] Planck collaboration, Planck 2018 results. VI. Cosmological parameters, arXiv: 1807.06209 [INSPIRE].

[29] F. Capozzi et al., Global constraints on absolute neutrino masses and their ordering, Phys. Rev. D 95 (2017) 096014 [arXiv: 1703. 04471] [INSPIRE].

[30] F. Simpson, R. Jimenez, C. Pena-Garay and L. Verde, Strong Bayesian evidence for the normal neutrino hierarchy, JCAP 06 (2017) 029 [arXiv: 1703.03425] [INSPIRE].

[31] P. F. De Salas et al., Neutrino mass ordering from oscillations and beyond: 2018 status and future prospects, Front. Astron. Space Sci. 5 (2018) 36 [arXiv:1806.11051].

[32] M. Singh, Testing texture two zero neutrino mass matrices under current experimental scenario, arXiv: 1909.01552 [INSPIRE].

[33] J. Alcaide, J. Salvado and A. Santamaria, Fitting flavour symmetries: the case of two-zero neutrino mass textures, JHEP 07 (2018) 164 [arXiv: 1806.06785] [INSPIRE].

[34] M. Singh, G. Ahuja and M. Gupta, Revisiting the texture zero neutrino mass matrices, PTEP 2016 (2016) 123B08 [arXiv: 1603.08083] [INSPIRE].

[35] S. Zhou, Update on two-zero textures of the Majorana neutrino mass matrix in light of recent

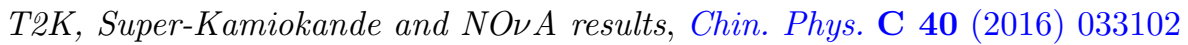
[arXiv: 1509.05300] [INSPIRE].

[36] T. Kitabayashi and M. Yasuè, Formulas for flavor neutrino masses and their application to texture two zeros, Phys. Rev. D 93 (2016) 053012 [arXiv:1512.00913] [INSPIRE].

[37] H. Fritzsch, Z.-z. Xing and S. Zhou, Two-zero textures of the Majorana neutrino mass matrix and current experimental tests, JHEP 09 (2011) 083 [arXiv:1108.4534] [INSPIRE].

[38] D. Meloni and G. Blankenburg, Fine-tuning and naturalness issues in the two-zero neutrino mass textures, Nucl. Phys. B 867 (2013) 749 [arXiv:1204.2706] [INSPIRE]. 
[39] S. Dev, S. Kumar, S. Verma and S. Gupta, Phenomenology of two-texture zero neutrino mass matrices, Phys. Rev. D 76 (2007) 013002 [hep-ph/0612102] [INSPIRE].

[40] W.-1. Guo and Z.-z. Xing, Implications of the KamLAND measurement on the lepton flavor mixing matrix and the neutrino mass matrix, Phys. Rev. D 67 (2003) 053002 [hep-ph/0212142] [INSPIRE].

[41] P.H. Frampton, S.L. Glashow and D. Marfatia, Zeroes of the neutrino mass matrix, Phys. Lett. B 536 (2002) 79 [hep-ph/0201008] [INSPIRE].

[42] R. Barbieri, T. Hambye and A. Romanino, Natural relations among physical observables in the neutrino mass matrix, JHEP 03 (2003) 017 [hep-ph/0302118] [INSPIRE].

[43] J.F. Nieves and P.B. Pal, Minimal rephasing invariant CP violating parameters with Dirac and Majorana fermions, Phys. Rev. D 36 (1987) 315 [INSPIRE].

[44] U. Sarkar and S.K. Singh, CP violation in neutrino mass matrix, Nucl. Phys. B 771 (2007) 28 [hep-ph/0608030] [INSPIRE].

[45] Particle Data Group collaboration, Review of particle physics, Phys. Rev. D 98 (2018) 030001 [INSPIRE]. 